Brake, J.H.M. te, Bouman, A.M., Gorter, R.C., Hoogstraten, J., Eijkman, M.A.J. Using the Maslach Burnout Inventory among dentists: burnout measurement and trends. Community Dentistry and Oral Epidemiology: 2008, 36(1), 69-75

\begin{tabular}{|l|l|}
\hline Postprint Version & 1.0 \\
\hline Journal website & $\underline{\text { http://www3.interscience.wiley.com/journal/119402075/abstract }}$ \\
\hline Pubmed link & $\underline{\text { http://www.ncbi.nlm.nih.gov/pubmed/18205642 }}$ \\
\hline DOI & $10.1111 / \mathrm{j} .1600-0528.2007 .00372 . x$ \\
\hline
\end{tabular}

This is a NIVEL certified Post Print, more info at http://www.nivel.eu

\title{
Using the Maslach Burnout Inventory among dentists: burnout measurement and trends.
}

\author{
J. H. M. Te Brake ${ }^{1}$, A. M. Bouman ${ }^{2}$, R. C. Gorter $^{1}$, J. HoOgSTRATEN ${ }^{1,2}$ AND M. A. J. EiJKMAN \\ ${ }^{1}$ Department of Social Dentistry and Dental Health Education, Academic Centre for Dentistry Amsterdam \\ (ACTA), Amsterdam, \\ ${ }^{2}$ Department of Psychological Methods, University of Amsterdam, Amsterdam, The Netherlands.
}

\begin{abstract}
Objectives: To extend our knowledge about the applicability of the Maslach Burnout Inventory (MBI) among dentists and to investigate trends in burnout risk among dental professionals. Methods: The structure of the MBI is examined among two independent, representative samples of dentists ( $\mathrm{n}=493$ and 497, respectively). Moreover, results are compared to those of other independently-practicing health professionals (i.e., general practitioners and physiotherapists). In addition, the percentage of dentists that are at risk for burnout are compared in samples from 1997, 2000, and 2001. Results: Correlations between the Emotional Exhaustion and Depersonalization subscales among dentists were higher than those found elsewhere. Additionally, a considerable increase in burnout risk was found since 1997. Conclusions: It was confirmed that the MBI can be considered a suitable instrument for use among dentists. It is argued that certain specific aspects of entrepreneur-like occupations are reflected in deviating responses to the MBI. Given the clear increase in burnout risk found, this topic should remain under investigation among dentists.
\end{abstract}

The measurement of burnout has long remained a controversial issue (1), which in part was the consequence of a lack of clear definition of the burnout concept [e.g., it has been described as a 'fuzzy set' of symptoms (Ref. 2, p. 76)]. Despite these difficulties, a large body of research on burnout has been conducted over the last decades, which primarily made use of the Maslach Burnout Inventory (MBI) (3). Burnout is defined as a threedimensional construct, characterized by emotional exhaustion (EE), depersonalization, and reduced personal accomplishment (PA) (3, 4). EE refers to feelings of being overextended, both emotionally and physically. Depersonalization (D) is characterized by negative, cynical attitudes and feelings about one's recipients. A reduced sense of PA refers to the negative evaluation of oneself, particularly in relation to job performance.

Entrepreneurs within the healthcare system, such as dentists, general practitioners, and physiotherapists, can be considered of special interest among the human services. Being a free entrepreneur, personal financial risk and high levels of autonomy characterize the working environment, but at the same time this freedom is confined by strong restrictions imposed by government and insurance companies. In addition, the dental work is characterized by isolated conditions (e.g., in almost three quarters of all Dutch dentists the mode of practice is solo - 5) and a strong reliance on technical skills, whereas in other human services professions colleagues or superiors are present, and counseling is more relevant than technical ability $(6,7)$. In earlier research (6) it was found that the Dutch version of the Maslach burnout inventory (MBI-NL) 
Brake, J.H.M. te, Bouman, A.M., Gorter, R.C., Hoogstraten, J., Eijkman, M.A.J. Using the Maslach Burnout Inventory among dentists: burnout measurement and trends. Community Dentistry and Oral Epidemiology: 2008, 36(1), 69-75

could be considered a suitable instrument for administration among Dutch dentists. Given the special position dentistry takes within health care, it is of interest not only to consolidate these findings but also to examine possible deviations that are specific for the field of dentistry.

A first aim of this study was to replicate and reaffirmthe applicability of the MBI among dentists.

Results of two independent samples of dentists (2001 and 2002) will be used to investigate the structure of the MBI-NL. In line with earlier findings by Gorter et al. (6), a three-factor structure is hypothesized to fit the data best. Results are compared to findings among other occupations among entrepreneurs within health care. Additionally, at present no data are available on the prevalence of burnout-risk among dentists. A second aim of this study was to determine the development of the level of risk among dentists. To this end, levels of burnout found in 1997 (8) will be compared to the findings in the abovementioned 2000 and 2001 studies, to attain a unique, longitudinal insight in the (in)stability of burnout-risk among dentists.

\section{MATERIALS AND METHODS}

\section{Participants and procedure}

Two large-scale surveys were performed among representative groups of dentists in the Netherlands. In the first study in 2000, a questionnaire was sent to a total of 885 dentists-general practitioner, all of which were approached previously in a related study conducted in 1997 (8). In 2001, a new group of 848 dentists was approached, making sure there was no overlap with respondents in the 2000 study. Care was given to ensure comparability with the general population of dentists in the Netherlands concerning the distribution of gender, age, and region. Furthermore, to heighten the power of gender comparisons, an extra sample (representative of age and region) of 95 female dentists was drawn. Comparison material among physiotherapists and general practitioners was obtained from Bakker et al. (9) and De Vries (10).

Where possible, findings among nonentrepreneurs were also used (based on research into physical and mental health care, geriatric care, disabled care, public welfare, and police/justice) (4).

\section{Materials}

In both studies, a Dutch translation of the MBI was used to measure burnout $(3,4,11)$. With the publication of a new manual (4) the Dutch version of the MBI was renamed Utrechtse Burnout Schaal (UBOS). Analogous to the original MBI, three versions of the UBOS were developed, distinguishing between contactual occupations (UBOS-C), teaching occupations (UBOS-L) and other, more general occupational groups (UBOS-A). In this study the UBOS-C was used, which is comparable with the MBI-human services survey (MBI-HSS) (3) and differs from the original MBI-HSS in that two items were deleted because they did not fit well in the proposed factor structure $(11,12)$. The remaining 20 items can be answered on a 7point Likert-scale, ranging from 0 ('never) to 6 ('every day'). Three subscale scores can be acquired - EE (eight items), D (five items), and PA (seven items). Examples of items from each subscale are: 'I feel emotionally drained from my work' (EE); 'I don't really care what happens to some patients' (D); and 'I deal very effectively with the problems of my patients’ (PA).

\section{Statistical analysis}

Internal consistency of the three subscales was assessed using Cronbach's alpha and interscale correlations were assessed by Pearson's correlations.

To address the first aim of the study, the dimensionality of burnout, six confirmatory factor analytic models (m0-m5) were analyzed using the LISREL 8.50 program (13). A null model (m0), in which all item scores are uncorrelated is necessary to create a baseline with which all other models can be compared. The second model (m1) was a onefactor model in which all items load on one common 'burnout' factor.

Because it was found that the EE and D scale show a high correlation and a two-factor model, in which EE and $\mathrm{D}$ are combined, showed a reasonable fit (6), such a model was also added (m2). The fourth model (m3) was an orthogonal three-factor model in which the three factors are not correlated with each other this is the original model (3). The fifth model (m4) was an oblique three-factor model in which the three factors are allowed to correlate. Finally, a second-order factor model (m5) in which the three factors load on one common fourth factor was included. In doing so, standardized factor loadings were obtained which give additional insight into the coherence between the three first-order factors (e.g., EE, D, and PA) and the second-order factor (e.g., 'burnout'). 
Brake, J.H.M. te, Bouman, A.M., Gorter, R.C., Hoogstraten, J., Eijkman, M.A.J. Using the Maslach Burnout Inventory among dentists: burnout measurement and trends. Community Dentistry and Oral Epidemiology: 2008, 36(1), 69-75

The goodness-of-fit of the models was determined using absolute and relative fit indices. The widely used absolute indices are supplemented with relative fit indices to draw better conclusions about the differences in goodness-of-fit between the different models. Absolute fit indices were the chi-square goodness-of-fit index with the accompanying degrees of freedom and the root mean square error of approximation (RMSEA). Relative fit indices are the Non-Normed Fit Index (NNFI), also called the Tucker Lewis Index (TLI), and the Comparative Fit Index (CFI). In contrast to the absolute indices, relative indices are independent of sample size and are therefore to be preferred (14). Furthermore, the NNFI is comparably more robust for sample size than both the RMSEA and the CFI (15). The fit of a model is good when the values of the NNFI and the CFI approach 1; a higher value represents a better fit. For these indices most studies consider a value of 0.90 or higher as indicative of a good fit $(10,16)$. For the RMSEA, values smaller than 0.08 imply a good fit.

Further possible improvements to the three-factor structure were investigated using explorative factor analysis (PCA, employing Varimax rotation) on both sets of data.

To address the second aim of the study, to compare the levels of burnout among dentists over time, results from the 1997 study (8) were compared to the results of the 2000 and 2001 studies.

First, mean levels of EE, D, and PA are computed.

The recommendations described in the 2000 UBOSmanual (4) were used to compute subscale scores.

As a result, computations differ from earlier scale scores in that no total scores are computed (i.e., summing all items scores) but mean scores (also taking into account the maximum number of items that may be missing per scale). Results of the 1997 study (8) are recalculated accordingly, to make comparison with the 2000 and 2001 studies possible.

As a further step, the level of burnout-risk was determined on all three points in time. Using the norm scores described in the UBOS manual (4), the three MBI-subscales can be combined to reach a conclusion on the relative risk for burnout. High scores on EE, combined with high scores on D or low scores on PA define a risk for burnout. By computing the percentage of dentists at risk in the 1997, 2000, and 2001 data, insight into possible trends can be attained.

\section{RESULTS}

\section{Response}

Of the 885 questionnaires that were sent in 2000, 22 proved undeliverable; 493 usable questionnaires were returned (response rate 57.1\%). A number of these dentists had participated in an intervention study, which had included two additional measurements $(8,17)$. Because in an earlier study $(17)$ it was found that these dentists' participation was likely to have an effect on the way they organized their working circumstances, this group was excluded from analyses in which a representative sample of the dental population was required (resulting in a total research group of 433 dentists). They were included, however, in the analyses on possible trends to ensure the 1997-2000 comparability. In 2001, 497 (58.6\%) of the 848 newly approached representative group of dentists returned a usable questionnaire. Of the 95 extra female dentists that were included, 63 responded (66.3\%). Except where indicated, these additional female dentists are not included in the analyses to ensure the generalizability with the Dutch dental population. Further descriptive characteristics are presented in Table 1; characteristics in 2000 did not significantly differ from the 2001 data. When compared with figures from the Dutch Dental Association (NMT) the sample characteristics of the 2000 study showed no significant differences. This further underscores the representativeness of the samples, although in the 2001 sample, female dentists are somewhat overrepresented.

\section{[TABLE 1] [TABLE 2]}

\section{Psychometric results}

Table 2 shows the internal consistencies and interscale correlations of the 2000 and 2001 studies when compared with the manual norm scores (4), physiotherapists (10), and general practitioners (9).

Results for the internal consistency indicate a high comparability between samples. The EE-D correlations in both studies were higher than those reported in the UBOS-C manual. However, they did correspond with the correlations found among physiotherapists and general practitioners. Both the EE-PA and the D-PA correlations among dentists showed some variations across studies; especially the higher correlation in 
Brake, J.H.M. te, Bouman, A.M., Gorter, R.C., Hoogstraten, J., Eijkman, M.A.J. Using the Maslach Burnout Inventory among dentists: burnout measurement and trends. Community Dentistry and Oral Epidemiology: 2008, 36(1), 69-75

2001 was noteworthy. These higher correlations in 2001 do correspond with the data found among general practitioners. Both in the study among physiotherapists and in the UBOS-C manual lower values are reported, consistent with the results from the 2000 study.

\section{MBI structure}

The goodness-of-fit indices are presented in Table 3.

For each of the six factor analytic models similar values were found in 2000 and in 2001. Compared with the other models, the oblique three-factor model (m4) showed the best fit; values of NNFI and CFI were closest to 1 and RMSEA was closest to 0 .

These results correspond with results found among physiotherapists. It should be noted, however, that in both studies the NNFI did not meet the criteria for a 'good fit', nor did the CFI in the 2000 study.

The goodness-of-fit indices for the $\mathrm{m} 5$ were identical to those of $\mathrm{m} 4$ because their mathematical structures were the same. However, the fitting of $\mathrm{m} 5$ did produce the standardized factor loadings of EE, $\mathrm{D}$, and PA on a common second-order factor ('burnout'). These factor loadings had corresponding patterns for both studies (2000 and 2001). The respective values of the factor loadings were $0.85,0.88$, and $) 0.43$ in 2000 , and $0.77,0.98$, and $) 0.57$ in 2001, for EE, D, and PA, respectively. These values are very similar to the factor loadings found among physiotherapists (EE 0.77, D 0.95, and PA )0.37).

Moreover, a corresponding pattern (e.g., high factor loadings for EE and D and lower loadings for PA) was found elsewhere (18), although it should be noted that in that study a slightly different version of the MBI was used.

Although it can be concluded that $\mathrm{m} 4$ displays the best fit to the current data, none of the theoretically proposed models $\mathrm{m} 0-\mathrm{m} 5$ indicate a clearly superior fit. In principle, alternative structures can exist that display a better fit. To explore this issue further, an explorative factor analysis on the 2000 and 2001 data, using Varimax rotation, was performed. This analysis indeed revealed an alternative four-factor structure in which two PA items (item 4: 'I can easily understand how my recipients feel about things' and item 7: 'I deal very effectively with the problems of my recipient') loaded on a separate fourth factor. The same analysis performed on the 2001 data did reveal the three-factor structure, although items 11 and 20 loaded on EE instead of D (as was theoretically proposed). Furthermore, item 20 had overall low loadings (EE 0.37, D 0.30, and PA )0.15).

As this latter finding corresponds with earlier results among dentists (6), it is possible that this constitutes a dental-specific deviation from the more general model of burnout. To explore this possibility, two alternative confirmatory models were fitted to establish if the goodness-of-fit of the best fitting model (the oblique three-factor, $\mathrm{m} 4$ ) could be improved. In the first alternative model, item 20 was excluded from the analysis and item 11 loaded on EE instead of D (m6). In the second alternative model, both items loaded on EE instead of $\mathrm{D}(\mathrm{m} 7)$. Results are shown in Table 3. Although in both studies v2 improved for $\mathrm{m} 6$ and, more notably, $\mathrm{m} 7$, goodness-of-fit did not meet critical standards. Therefore, $\mathrm{m} 4$ can be maintained as best fitting model.

\section{Burnout risk}

In Table 4 the mean MBI scores found among dentists are shown as found in 1997, 2000, and 2001 [as recommended in the UBOS manual (4), scores on the D scale are presented separately for men and women]. Each MBI subscale score relates to the frequency dentists experience certain feelings; a score of ' 0 ' means 'never', while ' 6 ' should be interpreted as 'every day'. The results in Table 4 should be interpreted accordingly: (i) dentists are 'now and then (once a month)' emotionally exhausted (score range 1.7-1.8); (ii) 'sporadically (a few times a year)' they have feelings of depersonalization (score range 1.21.3 for men, 1.0-1.1 for women); (iii) Dutch dentists '(very) often (at least once a week)' feel competent (score range 4.3-4.4).

Furthermore, no evident (positive or negative) trends emerge from the results in Table 4.

An alternative way to examine the risk of burnout is to use the definition as provided in the UBOS manual (4). Accordingly, a risk for burnout is defined as the combination of heightened levels of EE and D, with lowered levels of PA. Using this definition on all three samples, the results displayed in Table 5 were found. A clear negative trend now emerges, in which the percentage of dentists that are at a high level of risk increases between 1997 and 2001, which is mainly caused by the scores of male dentists. Male dentists also constitute the majority of the high-risk groups, while the opposite is true in the low-risk groups. 
Brake, J.H.M. te, Bouman, A.M., Gorter, R.C., Hoogstraten, J., Eijkman, M.A.J. Using the Maslach Burnout Inventory among dentists: burnout measurement and trends. Community Dentistry and Oral Epidemiology: 2008, 36(1), 69-75

When the norms for 'very high risk for burnout' are used (4), similar percentages are found over the years (3.1\%. 3.5\% and 3.2\% in 1997, 2000, and 2001, respectively).

\section{[TABLE 3, 4 AND 5]}

\section{DISCUSSION}

The purpose of this study was to elaborate on the use of the MBI-NL among dentists and examine possible trends over a 5-year period. A first aim was to further replicate the earlier findings about the usability of the MBI-NL among dentists. As hypothesized, both the results from a follow-up study, and a study among a new, independent sample of Dutch dentists, led to the conclusion that a three-factor model fitted the data best. In comparison with general practitioners and physiotherapists, some dental-specific deviations appeared to be present, although confirmative factor analyses revealed no significant improvement after these variations were taken into account. A second aim was to examine whether an increase or decrease in burnout levels could be found over the years. No such effects were found when comparing mean levels of EE, D, and PA. However, the relative percentage of burnout-risk among dentists indicates a substantial increase between the 1997, 2000, and 2001 samples. These results are discussed below.

The MBI was initially developed for research among human services, thus including the dental profession. However, being entrepreneurs, the working conditions of dentists also differ from the typical 'people work' setting in some important ways, as indicated at the beginning of the article. Considering this, the large interscale correlation between EE and D is of interest. The intercorrelation found among dentists was similar to those found among physiotherapists and general practitioners. In the ways it is akin to entrepreneurship, the latter occupation can be considered comparable to that of the dentist.

Furthermore, in an earlier study among dentists (6), a very strong relation between EE and D was also demonstrated (i.e., a model consisting of the combination of EE and D versus PA fitted the data better than a model of EE versus the combination of D and PA). In contrast, the EE-D intercorrelation reported among other healthcare professions (4) was substantially lower. Perhaps the large entanglement of EE and D is a specific phenomenon within healthcare professions that have a more 'solitary' daily practice. Regrettably, the current data provide no clear explanation for these results. Notwithstanding these considerations, the results generally confirm the proposed overall three-factor structure of burnout. Reliability coefficients and interscale correlations proved to be comparable not only to the values given in the manual, but also in comparison with other solo-oriented care-giving occupations such as physiotherapy and general practice. To this can be added that the results are strengthened by their confirmation in two independent samples. When the mean scores on EE, D, and PA are compared over the years, little or no differences are found, while at the same time the absolute levels on the subscales indicate relatively favorable burnoutlevels.

Such results could easily lead to the conclusion that continued attention for burnout among dentists is unwarranted. However, there are a number of reasons to declare such a conclusion inappropriate. For one, it is expected that high levels of burnout can lead dentists to prematurely stop active practice. Because the results are found among dentists that are actively at work in the practice, the results presented in Table 4 are likely to be positively biased. Second, it was found that when using the norm scores to examine the relative prevalence of burnout-risk, a substantial increase was found between 1997, 2000, and 2001. Finally, although the figures are derived from a group of dentists that is actively working in a dental practice, about $3 \%$ of these dentists indicate having very high levels of burnout.

With these findings it can be argued that issues concerning the use of the MBI in general should receive continued attention. As mentioned previously, the origin of the MBI can be questioned, but the generalizability among entrepreneur-like, more solo-oriented healthcare occupations even more so.

In future research, this distinction should not be neglected.

\section{ACKNOWLEDGMENTS}

This study was financially supported by Movir Insurance, Nieuwegein, The Netherlands. 
Brake, J.H.M. te, Bouman, A.M., Gorter, R.C., Hoogstraten, J., Eijkman, M.A.J. Using the Maslach Burnout Inventory among dentists: burnout measurement and trends. Community Dentistry and Oral Epidemiology: 2008, 36(1), 69-75

\section{REFERENCES}

1. Van Dierendonck D. Balancing the give and take. An equity approach to burnout. Dissertation. Utrecht: Universiteit Utrecht; 1997.

2. Burisch M. In search of theory: some ruminations on the nature and etiology of burnout. In: Schaufeli WB, Maslach C, Marek T, editors. Professional burnout. Recent developments in theory and research. Washington, DC: Taylor and Francis; 1993. p. 75-94.

3. Maslach C, Jackson SE, Leiter MP. MBI manual. $3^{\text {rd }}$ edn. Palo Alto, CA: Consulting Psychologists Press; 1996.

4. Schaufeli WB, Van Dierendonck D. Utrechtse burnout schaal. Handleiding (Dutch MBI-NL manual). Lisse, The Netherlands: Swets \& Zeitlinger B.V.; 2000.

5. College voor zorgverzekeringen. Tandheelkundige gezondheidszorg in Nederland. The Netherlands: Dental Health Care; 2001.

6. Gorter RC, Albrecht G, Hoogstraten J, Eijkman MAJ. Factorial validity of the Maslach Burnout Inventory - Dutch version (MBI-NL) among dentists. J Organ Behav 1999;20:209-17.

7. Gryskiewicz N, Buttner EH. Testing the robustness of the progressive phase model for a sample of entrepreneurs. Educ Psychol Meas 1992;52:747-51.

8. Gorter RC. Burnout among dentists: identification and prevention. Dissertation. Amsterdam, The Netherlands: University of Amsterdam; 2000.

9. Bakker AB, Schaufeli WB, Sixma HJ, Bosveld W. Burnout contagion among general practitioners. J Soc Clin Psychol 2001;20:82-98.

10. De Vries T. Burnout bij fysiotherapeuten met de verbijzondering manuele therapie. (Burnout among physio-manual-therapists). Dissertation. Amsterdam, The Netherlands: University of Amsterdam; 2001. p. 747-51.

11. Schaufeli WB, Van Dierendonck D. Maslach Burnout Inventory, Dutch version (MBI-NL). Provisional manual. Utrecht, The Netherlands: Utrecht University, Research School Psychology \& Health; 1995.

12. Schaufeli WB, Van Dierendonck D. The construct validity of two burnout measures. J Organ Behav 1993;14:631-47.

13. Jöreskog KG, Sörbom D. Lisrel: user's reference guide. Chicago, IL: Scientific Software International; 1996.

14. Bentler PM. Comparative fit indexes in structural models. Psychol Bull 1990;107:238-46.

15. Marsh HW, Balla JR, McDonald RP. Goodness-of-fit indexes in confirmatory factor analysis: the effect of sample size. Psychol Bull 1988;103:391-410.

16. Schaufeli WB, Salanova M, Gonza'lez-Roma' V, Bakker AB. The measurement of engagement and burnout: a two sample confirmatory factor analytic approach. J Happiness Studies 2002;3:71-92.

17. Te Brake JHM, Gorter RC, Hoogstraten J, Eijkman MAJ. Burnout intervention among Dutch dentists: long term effects. Eur J Oral Sci 2001;109:380-7.

18. Cordes CL, Dougherty TW, Blum M. Patterns of burnout among managers and professionals: a comparison of models. J Organ Behav 1997;18:685-701. 
Brake, J.H.M. te, Bouman, A.M., Gorter, R.C., Hoogstraten, J., Eijkman, M.A.J. Using the Maslach Burnout Inventory among dentists: burnout measurement and trends. Community Dentistry and Oral Epidemiology: 2008, 36(1), 69-75

\section{TABLES}

Table 1. Descriptive characteristics of the 2000 and 2001 samples

\begin{tabular}{lcc}
\hline & $\begin{array}{l}2000 \\
(n=433)\end{array}$ & $\begin{array}{l}2001 \\
(n=497)\end{array}$ \\
\hline $\begin{array}{l}\text { Gender (\%) } \\
\text { Male }\end{array}$ & \\
$\quad$ Female & 83.1 & 75.5 \\
Civil status (\%) & 16.9 & 24.5 \\
$\quad$ Single & & \\
$\quad$ Divorced & 4.3 & 4.3 \\
$\quad$ Married/cohabitation & 1.4 & 3.7 \\
$\quad$ Widow/widower & 0.1 & 91.6 \\
Mean age (SD) & $45.8(7.7)$ & 0.4 \\
Mean working & $31.7(7.6)$ & $34.6(9.0)$ \\
hours (SD) & & \\
Mean administration & $6.6(5.6)$ & $5.1(4.6)$ \\
hours (SD) & & \\
Number of patients per week $(\%)$ & \\
$\quad<20$ & 2.4 & 1.0 \\
$20-50$ & 8.9 & 12.6 \\
$50-75$ & 17.7 & 21.0 \\
$75-100$ & 23.9 & 17.9 \\
100-125 & 21.0 & 23.2 \\
$>125$ & 26.1 & 24.3 \\
\hline
\end{tabular}

Table 2. Internal consistency (diagonal) and interscale correlations of the UBOS-C subscales compared with the manual (4), Dutch physiotherapists (11), and Dutch general practitioners (9)

\begin{tabular}{|c|c|c|c|c|c|c|c|c|c|c|c|c|c|c|c|}
\hline & \multicolumn{3}{|c|}{ Study 2000} & \multicolumn{3}{|c|}{ Study 2001} & \multicolumn{3}{|c|}{ UBOS-C manual } & \multicolumn{3}{|c|}{ Physiotherapists } & \multicolumn{3}{|c|}{ General practitioners } \\
\hline & $\mathrm{EE}$ & $\mathrm{D}$ & PA & $\mathrm{EE}$ & $\mathrm{D}$ & PA & EE & $\mathrm{D}$ & PA & $\mathrm{EE}$ & $\mathrm{D}$ & PA & $\mathrm{EE}$ & $\mathrm{D}$ & PA \\
\hline EE & 0.89 & & & 0.90 & & & 0.87 & & & 0.91 & & & 0.90 & & \\
\hline D & 0.59 & 0.73 & & 0.59 & 0.70 & & 0.49 & 0.66 & & 0.59 & 0.71 & & 0.58 & 0.71 & \\
\hline PA & -0.29 & -0.28 & 0.79 & -0.36 & -0.44 & 0.83 & -0.20 & -0.30 & 0.79 & -0.23 & -0.28 & 0.80 & -0.44 & -0.41 & 0.71 \\
\hline
\end{tabular}

EE, emotional exhaustion; D, depersonalization; PA, personal accomplishment. 
Brake, J.H.M. te, Bouman, A.M., Gorter, R.C., Hoogstraten, J., Eijkman, M.A.J. Using the Maslach Burnout Inventory among dentists: burnout measurement and trends. Community Dentistry and Oral Epidemiology: 2008, 36(1), 69-75

Table 3. Factor analytic models of the UBOS-C for the studies in 2000 and 2001 compared with the UBOS-C manual (4) and Dutch physiotherapists (11)

\begin{tabular}{|c|c|c|c|c|c|}
\hline Model & $\chi^{2}$ & d.f. & RMSEA & NNFI & CFI \\
\hline \multicolumn{6}{|c|}{ Study 2000} \\
\hline $\mathrm{M}_{0}$ & 3619.94 & 190 & 0.310 & 0.00 & 0.00 \\
\hline $\mathrm{M}_{1}$ & 1222.81 & 170 & 0.140 & 0.66 & 0.69 \\
\hline $\mathrm{M}_{2}$ & 717.50 & 170 & 0.094 & 0.82 & 0.84 \\
\hline $\mathrm{M}_{3}$ & 797.08 & 170 & 0.090 & 0.80 & 0.82 \\
\hline $\mathrm{M}_{4}$ & 540.37 & 167 & 0.075 & 0.88 & 0.89 \\
\hline $\mathrm{M}_{5}$ & 540.37 & 167 & 0.075 & 0.88 & 0.89 \\
\hline $\mathrm{M}_{6}$ & 540.88 & 149 & 0.082 & 0.86 & 0.88 \\
\hline $\mathrm{M}_{7}$ & 581.89 & 167 & 0.080 & 0.86 & 0.88 \\
\hline \multicolumn{6}{|c|}{ Study 2001} \\
\hline $\mathrm{M}_{0}$ & 4663.65 & 190 & 0.340 & 0.00 & 0.00 \\
\hline $\mathrm{M}_{1}$ & 1529.20 & 170 & 0.160 & 0.66 & 0.70 \\
\hline $\mathrm{M}_{2}$ & 890.83 & 170 & 0.100 & 0.82 & 0.84 \\
\hline $\mathrm{M}_{3}$ & 991.33 & 170 & 0.096 & 0.79 & 0.82 \\
\hline $\mathrm{M}_{4}$ & 609.17 & 167 & 0.075 & 0.89 & 0.90 \\
\hline $\mathrm{M}_{5}$ & 609.17 & 167 & 0.075 & 0.89 & 0.90 \\
\hline $\mathrm{M}_{6}$ & 656.16 & 149 & 0.087 & 0.87 & 0.88 \\
\hline $\mathrm{M}_{7}$ & 705.71 & 167 & 0.086 & 0.86 & 0.88 \\
\hline \multicolumn{6}{|c|}{ Manual: total group } \\
\hline $\mathrm{M}_{0}$ & 86010.66 & 190 & a & 0.00 & 0.00 \\
\hline $\mathrm{M}_{2}$ & 37619.23 & 170 & a & 0.51 & 0.56 \\
\hline $\mathrm{M}_{4}$ & 7955.35 & 167 & $\mathrm{a}$ & 0.90 & 0.91 \\
\hline \multicolumn{6}{|c|}{ Physiotherapists } \\
\hline $\mathrm{M}_{0}$ & 10039.15 & 190 & a & 0.00 & a \\
\hline $\mathrm{M}_{1}$ & 2998.42 & 170 & a & 0.68 & a \\
\hline $\mathrm{M}_{2}$ & 1522.89 & 170 & a & 0.85 & a \\
\hline $\mathrm{M}_{3}$ & 1699.36 & 170 & $\mathrm{a}$ & 0.83 & a \\
\hline $\mathrm{M}_{4}$ & 1061.92 & 167 & a & 0.90 & a \\
\hline
\end{tabular}

$\chi^{2}$, chi-square goodness-of-fit index; d.f., degrees of freedom; RMSEA, root mean square error of approximation; NNFI, Non-Normed Fit Index; CFI, Comparative Fit Index; $\mathbf{M}_{0}$, null model; $\mathbf{M}_{1}$, one-factor model; $\mathbf{M}_{2}$, twofactor model (EE and D combined in one factor and PA as a second factor); $\mathrm{M}_{3}$, orthogonal three-factor model in which the three factors are not correlated with each other; $\mathrm{M}_{4}$, oblique three-factor model in which the three factors are correlated with each other; $\mathrm{M}_{5}$, second-order factor model; $\mathbf{M}_{6}$, an alternative for $\mathbf{M}_{4}$, with item 11 loading on EE and excluding item 20; $\mathbf{M}_{7}$, an alternative for $\mathrm{M}_{4}$, with both item 11 and item 20 loading on EE. ${ }^{\mathrm{a}}$ Values not reported in original studies. 
Brake, J.H.M. te, Bouman, A.M., Gorter, R.C., Hoogstraten, J., Eijkman, M.A.J. Using the Maslach Burnout Inventory among dentists: burnout measurement and trends. Community Dentistry and Oral Epidemiology: 2008, 36(1), 69-75

Table 4. Mean burnout scores (M) and standard deviation (SD) among Dutch dentists in 2001, 2000, and 1997

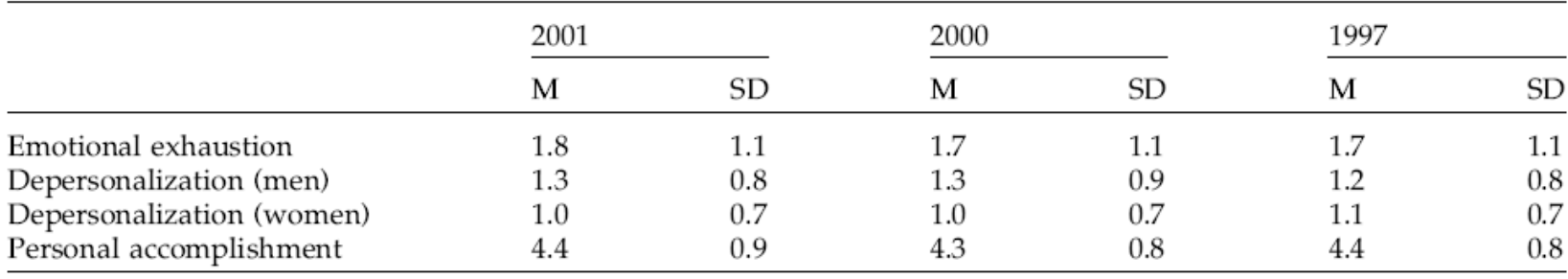

Table 5. Risk for burnout ${ }^{\mathrm{a}}$ : percentages for 1997, 2000, 2001, male and female dentists

\begin{tabular}{|c|c|c|c|c|c|c|c|c|c|}
\hline & \multicolumn{3}{|l|}{2001} & \multicolumn{3}{|l|}{2000} & \multicolumn{3}{|l|}{1997} \\
\hline & Male & Female & Total & Male & Female & Total & Male & Female & Total \\
\hline $\mathrm{n}$ & 372 & $184^{\mathrm{b}}$ & 495 & 402 & 80 & 483 & 575 & 109 & 689 \\
\hline Low risk & 5.7 & 8.4 & 6.4 & 9.0 & 12.5 & 9.5 & 6.8 & 8.8 & 7.3 \\
\hline Normal/average risk & 75.7 & 82.7 & 77.8 & 76.4 & 76.3 & 76.4 & 81.2 & 83.2 & 81.4 \\
\hline High risk & 18.5 & 8.9 & 15.8 & 14.7 & 11.3 & 14.1 & 12.0 & 8.0 & 11.3 \\
\hline
\end{tabular}

${ }^{a}$ The low-risk group is defined by dentists that have a combination of (very) low scores on EE and D, and (very) high scores on PA. The high-risk group is defined by dentists that have a (very) high score on EE, in combination with a (very) high score on D or a (very) low score on PA. Dentists that are not categorized as either low risk or high risk are classified as 'normal/average risk'.

${ }^{\mathrm{b}}$ Number of cases includes an extra sample of female dentists $(n=63)$. 